\title{
ЗАБОЛЕВАЕМОСТЬ И СМЕРТНОСТЬ ОТ COVID-19. ПРОБЛЕМА СОПОСТАВИМОСТИ ДАННЫХ
}

\author{
ИННА ДАНИЛОВА
}

\begin{abstract}
Числа заболеваний и смертей от COVID-19 сегодня привлекают внимание всего мира. Различные вебресурсы публикуют эти данные по странам и их регионам. Данные доступны всем желающим и обновляются практически в режиме реального времени. Оперативный сбор данных о заболеваниях и смертях и их открытость, несомненно, дают значительные преимущества в борьбе с эпидемией, прогнозировании ее развития и планировании мер по сдерживанию инфекции. Важный аспект анализа эпидемии COVID-19 - сравнение стран и территорий по уровню заболеваемости, летальности и смертности. Учитывая возможности, открывающиеся сегодня для анализа данных, крайне важно понимать, согласно каким критериям эти данные собираются.

В данной статье обсуждаются ключевые аспекты сбора данных о числе заболеваний и смертей от COVID-19 и поднимается вопрос о сопоставимости этих данных между странами. Показано, что страны существенно отличаются в подходах к учету заболеваний и смертей от COVID-19. На сопоставимость данных между странами могут, в частности, влиять обеспеченность тестами, принятые в стране критерии тестирования на вирус (как прижизненного, так $u$ посмертного), подходы к определению причины смерти и учета COVID-19-смертей в случае смерти людей с предшествуюшими хроническими заболеваниями. Разные подходы к учету заболеваний и смертей от COVID-19 между странами, а также их изменения во времени представляют существенные ограничения для возможностей текущей оценки развития эпидемии. Эти ограничения должны обязательно приниматься во внимание при анализе заболеваемости и смертности от COVID-19, так как их игнорирование может существенно исказить наше понимание распространения эпидемии на различных территориях.
\end{abstract}

Ключевые слова: COVID-19, сбор данных, причины смерти, статистика заболеваемости, статистика смертности.

\section{ВВЕДЕНИЕ. КАКИЕ ДАННЫЕ НАМ ДОСТУПНЫ?}

31 декабря 2019 г. Китай сообщил во Всемирную организацию здравоохранения (ВОЗ) о том, что в городе Ухань зафиксирована вспышка случаев пневмонии, вызванной неизвестным возбудителем. Уже 7 января 2020 г. китайские специалисты идентифицировали вызывавший эту пневмонию вирус. Это оказался новый, до этого не известный вирус семейства коронавирусов. Сначала он получил временное название 2019-nCov, сегодня мы его знаем как вирус под названием SARS-CoV-2. Именно этот вирус вызывает болезнь, которая получила название COVID-19. Спустя еще два месяца, 11 марта 2020 г., ВОЗ объявила пандемию - признание того, что болезнь уже распространилась глобально и охватила все континенты (World Health Organization 2020a). Это уже вторая пандемия, которую ВОЗ объявляет в XXI веке.

\footnotetext{
ИННА АНДРЕЕВНА ДАНИЛОВА (danilova@demogr.mpg.de), ИНСТИТУТ ДЕМОГРАФИЧЕСКИХ ИССЛЕДОВАНИЙ ОБЩЕСТВА МАКСА ПЛАНКА, ГЕРМАНИЯ.

СТАТЬЯ НАПИСАНА НА ОСНОВЕ ДОКЛАДА, ПРЕДСТАВЛЕННОГО НА ВЕБИНАРЕ МЕЖДУНАРОДНОЙ ЛАБОРАТОРИИ ИССЛЕДОВАНИЙ НАСЕЛЕНИЯ И ЗДОРОВЬЯ НИУ ВШЭ “COVID-19: КОЛИЧЕСТВЕННАЯ ОЦЕНКА ПАНДЕМИИ И ЕЕ ВОЗДЕЙСТВИЯ НА УРОВЕНЬ СМЕРТНОСТИ” 9 АПРЕЛЯ 2020 ГОДА. ВИДЕОЗАПИСЬ ДОКЛАДА ДОСТУПНА ПО ССЫЛКЕ: HTTPS://DEMOGR.HSE.RU/NEWS/357669566.HTML.
} 
Предыдущая - пандемия гриппа А серотипа (H1N1) - была объявлена в 2009 г. Это был тот же серотип гриппа А, который вызвал в свое время Испанку, но другой его штамм. Нынешняя пандемия примечательна тем, что это первая известная пандемия, вызванная коронавирусом (World Health Organization 2020a). Наверное, она еще более примечательна теми глобальными карантинными мерами, которые сегодня применяют правительства очень многих стран. И еще одна уникальная особенность нынешней пандемии - возможность наблюдать практически в онлайн-режиме за тем, как изменяются числа заболевших и умерших. Ежедневно обновляемые и легко доступные массивы данных - это то, чего невозможно было себе представить еще, может быть, 10-15 лет назад.

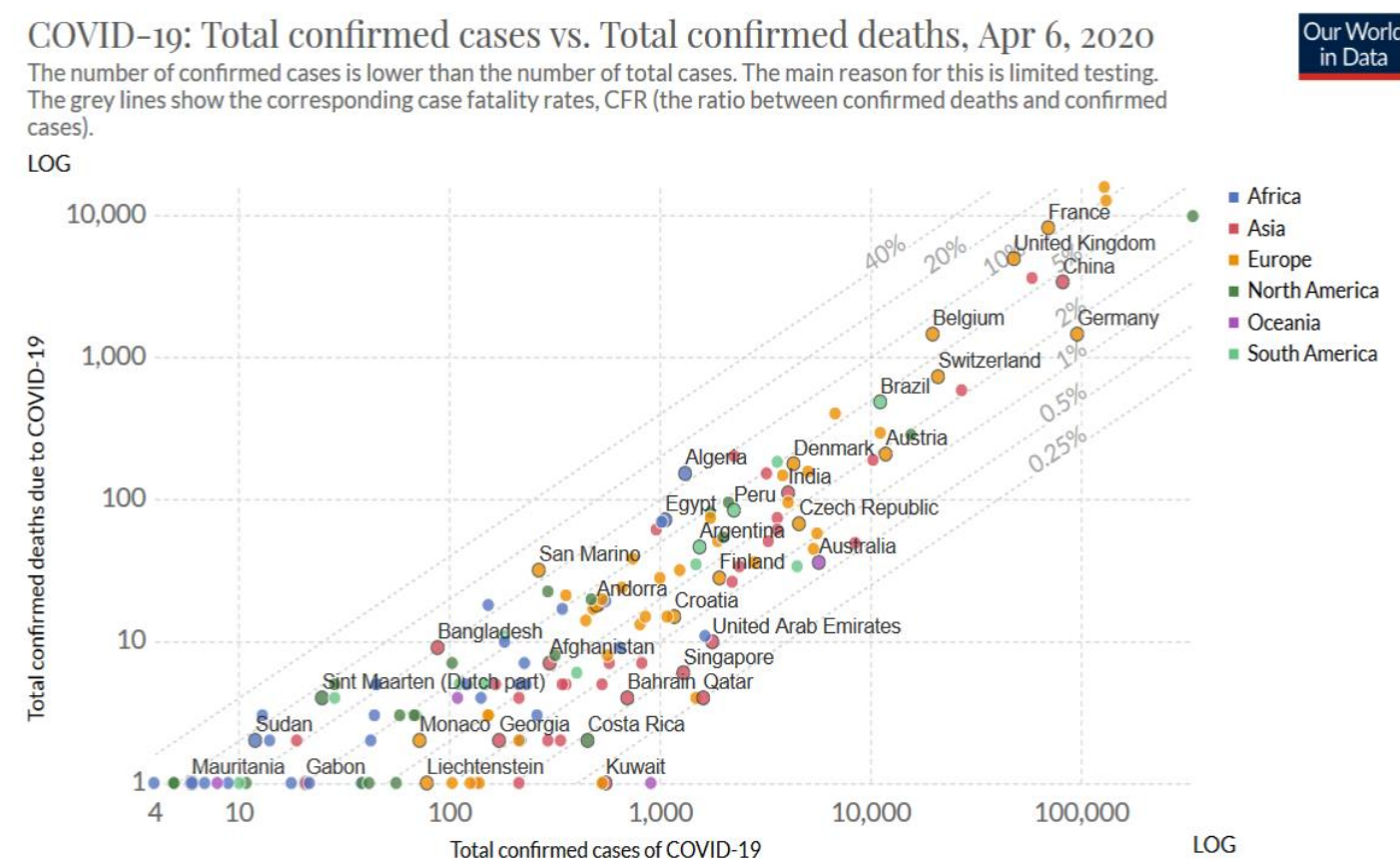

\section{Рисунок 1. Отношение числа умерших к числу заболевших (Case Fatality Rate) по странам мира на 6 апреля 2020 г. (логарифмическая шкала)}

Источник: Our World in Data. URL:https://ourworldindata.org/coronavirus

Есть множество сайтов, публикующих статистику по COVID-19. Например, на сайте института Джона Хопкинса представлена карта мира, где можно отслеживать число заболевших и умерших по странам ${ }^{1}$. Есть подобные карты для территорий внутри стран. Яндекс строит такую карту для регионов России ${ }^{2}$. Есть сайты, где данные представлены в виде различных табличек, показывающих ежедневный прирост числа заразившихся и умерших по сравнению со вчерашним днем (например, сайт Worldometer ${ }^{3}$ ).

Что мы видим, глядя на эти данные? Во-первых - это то, что страны очень сильно отличаются по числу заболеваний и числу смертей. Для примера рассмотрим график,

\footnotetext{
${ }^{1}$ URL:https://coronavirus.jhu.edu/map.html

${ }^{2}$ URL:https://yandex.ru/maps/COVID19

${ }^{3}$ URL: https://www.worldometers.info/coronavirus/ 
заимствованный с сервиса Our World in Data ${ }^{4}$ (рисунок 1). По оси X представлено общее число подтвержденных случаев инфекции Covid-19, а по оси Y - общее число смертей. К сожалению, данные представлены в абсолютных числах, а не в расчете на душу населения. Серые диагонали на графике - это интересующий нас показатель Case Fatality Rate: отношение числа умерших к числу заболевших. Мы видим, что страны очень сильно различаются по этому показателю, даже страны, где эпидемия началась примерно в одно и то же время и которые, казалось бы, находятся на одном уровне экономического развития и уровне развития здравоохранения. Сравним, например, две соседние страны - Германию и Францию. В Германии Case Fatality Rate менеe 2\%, а во Франции он уже больше 10\% (на 6 апреля 2020 г.) - разница огромная.

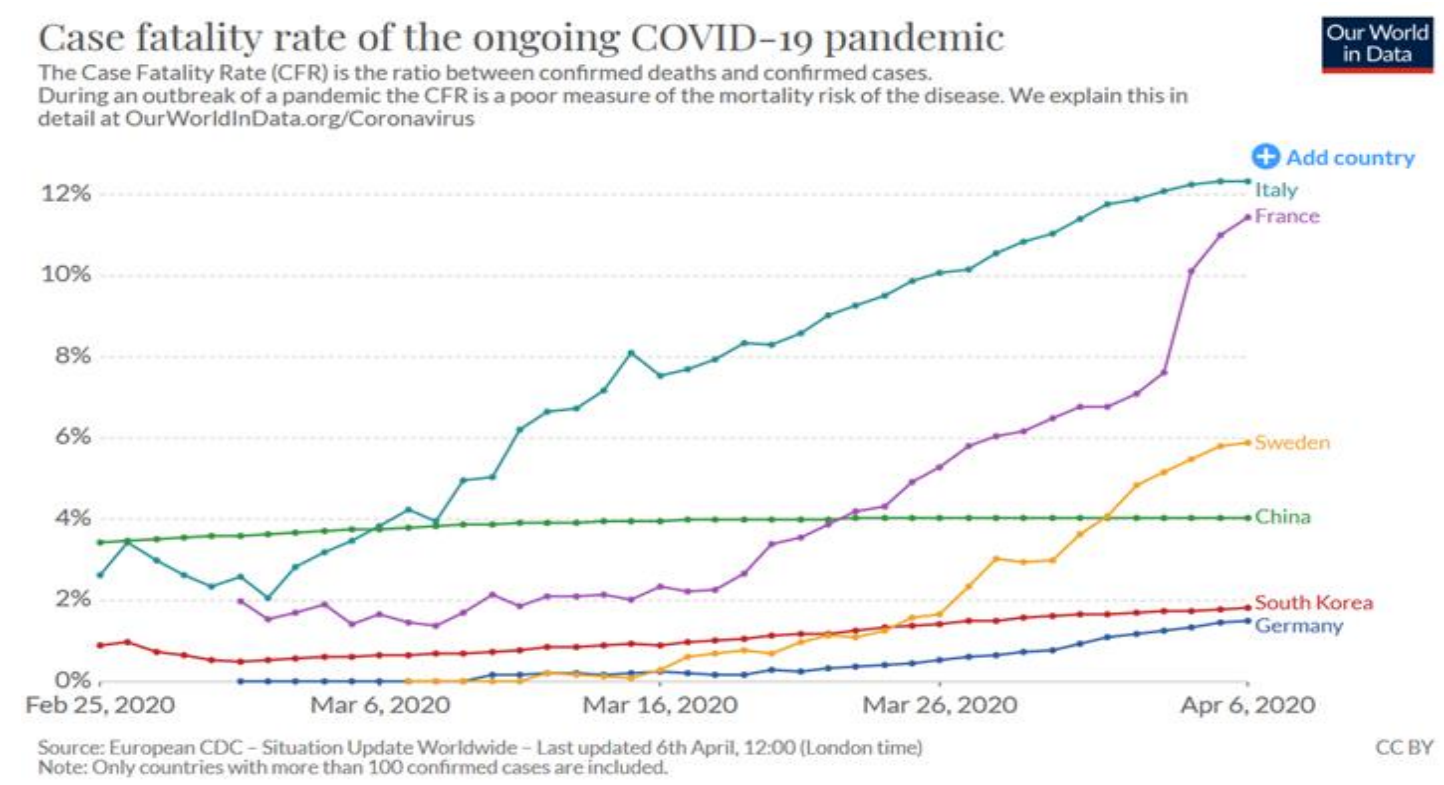

\section{Рисунок 2. Изменения показателя Case Fatality Rate по странам с 25 февраля по 6 апреля 2020 г.}

Источник: Our World in Data. URL:https://ourworldindata.org/coronavirus

Показатель Case Fatality Rate меняется также во времени. С того же сайта Our World in Data взяты графики динамики показателя Case Fatality Rate для некоторых стран (рисунок 2). Мы видим, что, например, в Италии, Франции, Швеции показатель Case Fatality Rate быстро растёт. На это могут влиять как естественный процесс развития эпидемии, так и изменения в практиках учета. Последнее хорошо заметно на примере Франции, где виден очень резкий скачок Case Fatality Rate в апреле. Это следствие того, что с апреля во Франции в статистику начали включать смерти в домах престарелых и других медико-социальных учреждениях (Santé publique France 2020). До этого отчетность шла только по тем медицинским свидетельствам о смерти, которые были выданы в учреждениях системы здравоохранения, а медико-социальные учреждения во Франции относятся к другой системе. Когда стали собирать более полные данные, показатель Case Fatality Rate во Франции резко вырос.

\footnotetext{
${ }^{4}$ URL:https://ourworldindata.org/coronavirus
} 
Каковы ключевые показатели распространения инфекции, которые исследователи сегодня пытаются оценить?

Во-первых, конечно, важно знать общее число инфицированных в населении и, стало быть, сколько людей ещё не инфицировано. Как эти числа меняются во времени? Следующий важный показатель - это летальность, т. е. сколько из тех, кто инфицирован, умирает. Как летальность зависит от возраста и от пола заболевших? И наконец, показатель, наверное, наиболее интересный с точки зрения демографического анализа: как вследствие вспыхнувшей эпидемии изменится смертность? Сколько дополнительных смертей она принесет? Как это повлияет на наблюдавшиеся до сих пор тренды смертности и продолжительности жизни?

Чтобы ответить на эти вопросы и нужны все указанные показатели и важны соотношения между ними. Показатели же, которые мы можем анализировать, в свою очередь зависят от того, с какой полнотой выявляются случаи инфицирования COVID-19, как они диагностируются, как при определении причины смерти устанавливается, что смерть произошла именно от COVID-19, а не от чего-то ещё.

Сейчас имеется бесчисленное множество сайтов, на которых можно увидеть, сколько человек заболело и сколько человек умерло, но при этом мы не знаем, как конкретно собираются приводимые на них данные, на каких критериях они основываются, как эти критерии различаются по странам.

\section{КОГО ВКЛЮЧАЮТ В СТАТИСТИКУ ЗАБОЛЕВАЕМОСТИ COVID-19?}

Начнем с самой простой характеристики: что такое вообще подтверждённые случаи COVID-19. Есть официальное определение BO3, которое эта организация использует для того, чтобы следить за пандемией. Теоретически все страны должны отчитываться, исходя из этого определения. Подтверждённый случай - это случай «с лабораторным подтверждением наличия инфекции COVID-19 независимо от клинических проявлений и симптомов заболевания» (World Health Organization 2020b). Насколько можно судить, те числа заболевших, которые публикуют страны, соответствуют именно этому определению и, таким образом, основаны на подтвержденных положительных тестах. Единственное исключение, которое нам удалось найти, это американский штат Колорадо. На сайте правительства штата публикуются данные о числе заболевших. При этом указывается, что в это число включены как люди, у которых был обнаружен положительный результат при тестировании, так и те, кого не тестировали, но для кого эпидемиологическое расследование определило высокую вероятность быть инфицированным, потому что у человека имеются симптомы COVID-19 и был близкий контакт с кем-то, у кого тестирование показало положительный результат. При этом оговаривается, что число не протестированных, но включённых в статистику очень мало 5 .

\footnotetext{
${ }^{5}$ URL:https://COVID19.colorado.gov/data/case-data 


\section{КОГО ТЕСТИРУЮТ НА COVID-19?}

Итак, согласно определению BO3, чтобы случай COVID-19 был учтен в статистике, необходимо подтвердить его положительным тестом на присутствие вируса. Здесь возникает вопрос: кому делают тесты? Каковы критерии того, должен или не должен человек пройти тестирование? Разница в ответах на этот вопрос между странами довольно велика. От чего на практике зависит, кому будут сделаны тесты?

В первую очередь это зависит от доступных в стране или регионе лабораторных мощностей тестирования. С того момента как вирус вообще был идентифицирован, прошло всего три месяца. После того, как вирус идентифицировали, необходимо было разработать тесты, которые бы его обнаруживали, опробовать и одобрить их на уровне правительств или их органов здравоохранения. Нужно было начать производить эти тесты, подготовить лаборатории, чтобы они могли сделать анализ, нарастить эти мощности там, где их было недостаточно, и так далее. Все это нужно было сделать в течение короткого промежутка времени. Некоторые страны справились с этими задачами хорошо. Так, например, в Южной Kopeе, Сингапуре, Австралии начали тестировать людей на COVID-19 уже в конце января и тестировать довольно широко. Другие страны сделали это с задержкой, и там вплоть до марта тестирования практически не было. Например, в США первоначально произведенные тесты были плохого качества, задержка также была связана с длительным ожиданием одобрения тестов от Управления по санитарному надзору за качеством пищевых продуктов и медикаментов США. Разрыв в возможностях тестирования сохраняется между странами и сегодня. Неясно, насколько готовы к массовому тестированию бедные страны Африки или Латинской Америки, многие азиатские страны, способны ли они организовать тестирование в достаточных масштабах.

Следующая проблема возникла в марте из-за того, что когда тестов стало выполняться очень много, возникла нехватка реагентов для тестирования. Об этом пишет BO3 21 марта (World Health Organization 2020c), но правительства некоторых стран об этом стали говорить еще раньше. Возросшее тестирование на Covid-19 привело к нехватке тестовых реагентов в мировом масштабе, а также к нехватке реагентов другой молекулярной диагностики. В этих условиях возникла проблема приоритетов: кому тесты делаются в первую очередь и на основании каких критериев.

Имеется официальная рекомендация ВО3 по стратегиям лабораторного тестирования (World Health Organization 2020c). В этой рекомендации все страны разделены на 4 группы: 1) страны, где ещё нет зафиксированных случаев инфекции; 2) страны, где пока отмечены единичные спорадические случаи; 3) страны, где уже существуют локализованные кластеры случаев; 4) страны, где наблюдается передача инфекции внутри местных сообществ. Первые три группы стран должны, согласно рекомендациям ВОЗ, тестировать каждый подозрительный случай на COVID-19. К подозрительным относится не каждый случай, когда у человека есть температура, болит горло или он кашляет. Согласно определению BO3, подозрительным на COVID-19 считается человек, у которого есть острое респираторное заболевание и который приехал из какого-то места, где есть вспышка заболеваемости Covid-19 (места, где заболевание передается внутри страны или района). Подозрительным на Covid-19 также считается человек с острым респираторным 
заболеванием, у которого был контакт с человеком с подтвержденным заражением COVID-19. Если у человека не было ни истории путешествий, ни подтвержденного контакта с больным, то для того, чтобы считаться подозрительным случаем и быть протестированным, согласно рекомендациям ВОЗ, он должен испытывать серьезное острое респираторное заболевание (OP3), требующее госпитализации, и не должен иметь какогото альтернативного диагноза, объясняющего его симптомы (World Health Organization 2020c). Все это относится к странам, где вспышки ещё локализованы и малы.

Что же касается стран, уже охваченных инфекцией, где инфекция уже не только завозная, не локализована на каких-то ограниченных территориях, а достаточно широко распространена и активно передаётся внутри страны, то, как предупреждает ВОЗ, в таких странах можно ожидать дефицита тестов и нужно вводить приоритеты, кого тестировать. В том же документе ВОЗ отмечается, что так как для вируса не имеют значения административные границы, можно ожидать, что даже внутри одной страны могут понадобиться различные стратегии тестирования (World Health Organization 2020c). Стратегии тестирования будут в свою очередь влиять на выявляемость и на соотношение выявленных и не выявленных инфицированных. Это соотношение зависит от того, тестируется ли в данной стране каждый подозрительный случай или там вводятся какие-то критерии отбора и, например, тестируются только те, кому нужна госпитализация или те, кто серьезно болен, или по каким-то еще критериям.

Сейчас в большинстве стран есть установленные критерии, согласно которым должны делаться тесты, и решения о том, кто соответствует или не соответствует этим критериям и кого надо тестировать, принимают врачи. Существуют определенные методические указания, инструкции для медицинских работников, как определить, кого следует тестировать на COVID-19, а кого нет. Но есть и такие страны, где человек может пройти тестирование, если у него просто возникло такое желание, не консультируясь с доктором.

Пример такой страны - Исландия. В Исландии на 8 апреля было сделано уже более 30 тыс. тестов ${ }^{6}$. Для населения Исландии это огромное число, на данный момент это одна из самых протестированных наций мира. Национальный университетский госпиталь Исландии использует очень широкие критерии для тестирования на COVID-19, основания для него дает довольно широкий круг симптомов. Кроме того, в Исландии можно добровольно пройти тест в рамках исследований, которые проводит лаборатория deCode Genetics (Gudbjartsson et al. 2020). Человек может просто на сайте лаборатории подать заявку, что он хочет быть протестирован на Covid-19, и через какое-то время ему сделают тест независимо от того, есть у него симптомы или нет.

Нечто подобное имеется и в России. Коммерческие лаборатории в крупных городах (Москве, Санкт-Петербурге, Екатеринбурге) предлагают тестирование на SARS-CoV-2. Если в такой лаборатории тест оказывается положительным или сомнительным, то его отправляют в лабораторию Роспотребнадзора, там его перепроверяют и подтверждают или не подтверждают случай COVID-19. В США тоже была попытка организовать станции,

\footnotetext{
${ }^{6} \mathrm{URL}:$ https://www.covid.is/data 
куда можно было приехать и, не выходя из машины, пройти тестирование, сдать мазок, причем в некоторых штатах для этого требовалось направление врача, в других достаточно было сообщить о симптомах, а лаборант сам решал, проводить тестирование или нет. Но впоследствии, насколько нам известно, эти станции в США были закрыты, потому что у них также обнаружилась нехватка тестов и их стали экономить для тех, кому они нужнее.

Вопрос о тестировании достаточно важен, потому что от него зависит оценка как числа заболевших, так и летальности заболевания. В Исландии, где тестируют очень широко и в том числе людей без симптомов, уровень выявляемости будет совсем другим, чем в странах, где тестирование предполагает более или менее жесткие критерии. Соответственно, в Исландии в числе заболевших будет гораздо больше людей без симптомов или с мягкими симптомами и в то же время будет более низкая летальность.

Критерии тестирования могут меняться во времени. Это хорошо видно на примере Германии. Здесь Институт Роберта Коха на своём сайте публикует инструкции для врачей в виде схем, где врач может по стрелочкам пройтись и решить, нужно человеку делать тест на COVID-19 или нет. Например, в схеме от 21 января (Robert Koch Institut 2020а) указано, что тестированию (после консультации с лабораторией в Берлине) на COVID-19 подлежали: 1) пациенты с клиническими радиологическими или гистопатологическими признаками пневмонии, приехавшие из районов повышенного риска (в скобках конкретно указывалось: Китай, Ухань, провинция Хубэй); 2) пациенты, у которых острое респираторное сочеталось с контактом с подтвержденным больным COVID-19.

Через два дня эти критерии немного изменились (Robert Koch Institut 2020b). Для первой группы пациентов (приехавших из районов повышенного риска) в схеме указывалась уже не пневмония, а просто острая инфекция нижних дыхательных путей. Также «районы повышенного риска» в обновленной схеме были без уточнения конкретных названий, смотреть актуальный список этих территорий предлагалось на сайте Института Роберта Коха.

10 февраля показания к тестированию были уже намного шире (Robert Koch Institut 2020c). Теперь для приехавших из районов повышенного риска было достаточно просто иметь острые респираторные симптомы. Если у человека был подтвержденный контакт с COVID-19, то тестировать полагалось как при наличии острых респираторных симптомов, так и при наличии неспецифических общих симптомов.

В инструкции от 26 февраля (Robert Koch Institut 2020d) люди с симптомами OР3, вернувшиеся не только с «территорий повышенного риска», но и с «территорий, с зафиксированными случаями COVID-19» (список этих территорий также регулярно обновлялся на сайте Института Роберта Коха), могли быть протестированы на вирус. Люди с подтвержденным контактом с больным COVID-19 по-прежнему должны были быть протестированы при наличии симптомов ОРЗ или неспецифичных общих симптомов. Но даже если у человека не было контакта с носителями COVID-19 и он ниоткуда не приезжал, но у него наблюдалось клиническое и радиологическое подтверждение вирусной пневмонии без альтернативного диагноза, он тоже может быть протестирован на COVID19. 
Ещё через месяц, 24 марта (Robert Koch Institut 2020e), критерии снова меняются. Что изменилось? Во-первых, исчезли ограничительные указания на регионы, из которых можно было завезти инфекцию. Вирус уже распространяется внутри Германии, и не имело большого значения, кто откуда приехал. При контакте с больным COVID-19 исчезло указание на неспецифические общие симптомы. Теперь даже в случае подтвержденного контакта с носителем COVID-19 требуется всё-таки, чтобы у человека были симптомы острого респираторного заболевания. Тестированию также подлежат случаи с клиническими или радиологическими признаками пневмонии, особенно связанные с увеличившимся числом пневмоний в домах престарелых или больницах, но также и те, для которых не установлено альтернативного диагноза. Те, у кого просто симптомы острого респираторного заболевания, тестируются на вирус, если они работают в домах престарелых, учреждениях здравоохранения или относятся к группе риска. Если человек с острыми респираторными симптомами не попадает под эти критерии, то в инструкции для врачей указано, что он может быть протестирован только при наличии достаточного количества тестов.

\section{НЕДООЦЕНКА ЗАБОЛЕВАЕМОСТИ COVID-19 СРЕДИ МОЛОДОГО НАСЕЛЕНИЯ ВЫШЕ, ЧЕМ ДЛЯ ПОЖИЛЫХ?}

Во многих странах приняты такие критерии, что тесты в первую очередь делаются наиболее уязвимым группам населения, в том числе людям с хроническими заболеваниями. Это чаще всего пожилые люди. Соответственно, можно ожидать, что выявляемость вируса среди пожилых будет выше, чем среди более молодых возрастных групп. Это повлияет и на достоверность оценок летальности в разных возрастах.

На рисунке 3 представлены две группы стран. В верхнем ряду Италия, Испания и Бельгия. Эти страны находятся среди лидеров как по заболеваемости и смертности, так и по показателю Case Fatality Rate от COVID-19 (по данным на 9 апреля). И мы знаем, что в этих странах тесты делаются весьма ограничено. Для того, чтобы человек был протестирован на COVID-19, он либо должен быть госпитализирован, либо у него должны быть уже довольно серьёзные симптомы заболевания.

Синие столбики на графике - это распределение подтвержденных случаев COVID-19 по возрасту, начиная с 20 лет. Оранжевые - распределение по возрасту всего населения. Мы видим, что в странах верхнего ряда распределение числа заболевших сильно сдвинуто к пожилым возрастам, и в этом смысле оно сильно отличается от распределения населения по возрасту. Например, в Италии доля заболевших, которая приходится на возраст 90 лет и старше, в 3 раза выше, чем доля населения в этом возрасте. Но в странах с существенно более высоким уровнем тестирования (Исландия, Австралия ${ }^{7}$, Южная Корея) структура выявленных случаев гораздо больше соответствует общей возрастной структуре населения, и сильного превышения доли заболевших в старших возрастах по сравнению с их долей в общей структуре населения нет.

\footnotetext{
${ }^{7}$ Представлен самый пораженный штат - Новый Южный Уэльс. 


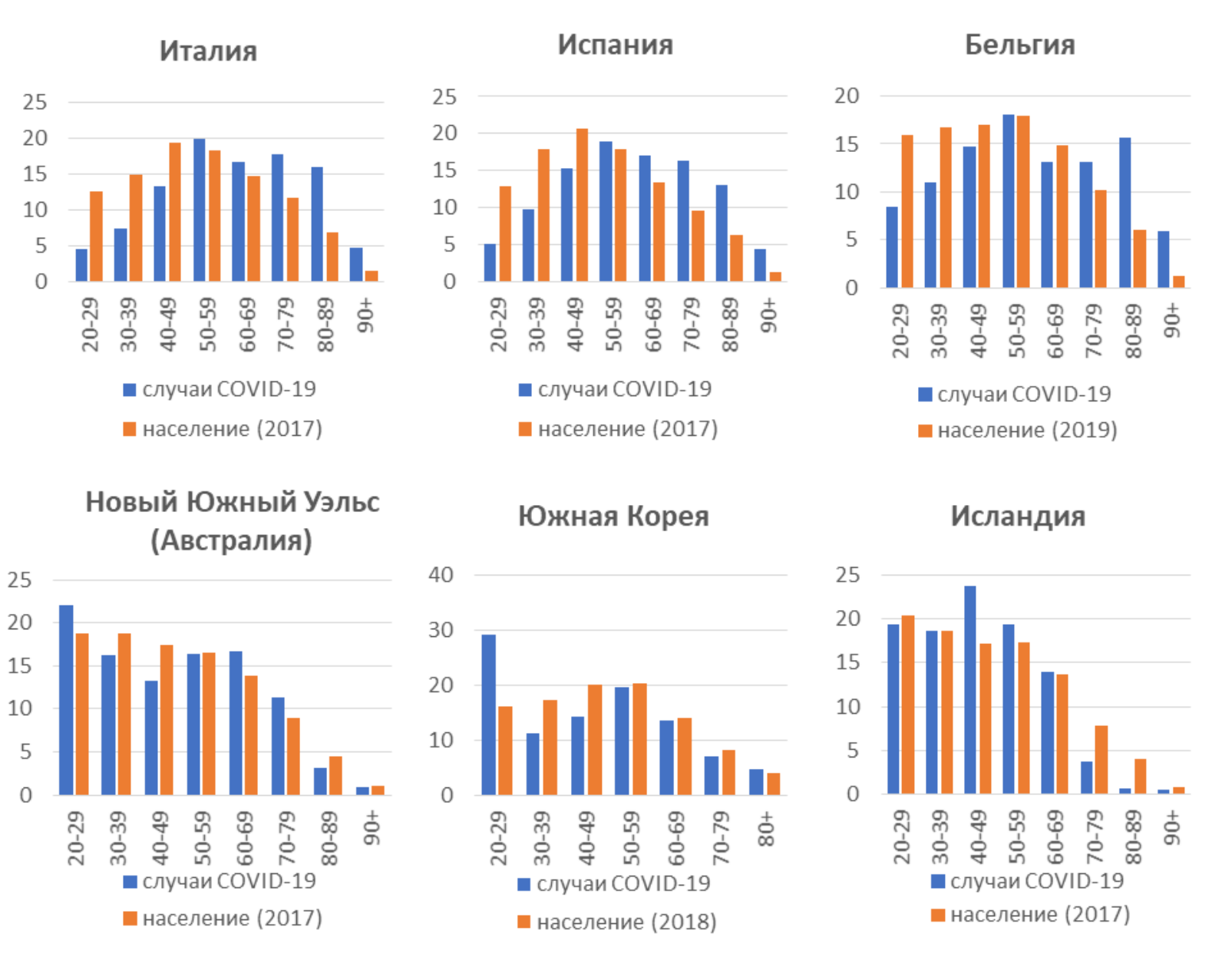

\section{Рисунок 3. Возрастная структура населения и возрастная структура заболеваний COVID-19 в некоторых странах, \%}

Источники: Оченки численности населения: Италия, Испания, Бельгия, Исландия, Южная Корея (Human Mortality Database) ${ }^{8}$, Южный Новый Уэльс (Australian Human Mortality Database) ${ }^{9}$. Заболеваемость COVID-19: Италия ${ }^{10}$, Испания $^{11}$, Бельгия ${ }^{12}$, Исландия ${ }^{13}$, Новый Южный Уэльс ${\text { (Австралия })^{14} \text {, Южная Корея }}^{15}$.

Можно искать какие-то другие объяснения особенностей возрастного распределения случаев COVID-19 по странам. Например, в том, что в Италии, Испании, Бельгии больше людей живет в домах престарелых (именно дома престарелых стали очагами инфекции во многих странах), что в южной Европе (в частности, Италии и Испании) люди чаще живут

\footnotetext{
${ }^{8}$ Human Mortality Database. URL:https://www.mortality.org/

${ }^{9}$ Australian Human Mortality Database. URL:https://demography.cass.anu.edu.au/research/australian-humanmortality-database

${ }^{10}$ URL:https://www.epicentro.iss.it/coronavirus/bollettino/Bollettino-sorveglianza-integrata-COVID-19_6-aprile-

2020.pdf

${ }^{11}$ URL:https://www.mscbs.gob.es/profesionales/saludPublica/ccayes/alertasActual/nCov-

China/documentos/Actualizacion_69_COVID-19.pdf

12 URL:https://covid-19.sciensano.be/sites/default/files/Covid19/COVID-19_Daily\%20report_20200408\%20-

$\% 20$ FR.pdf

${ }^{13}$ URL:https://www.covid.is/data

${ }^{14}$ URL:https://www.health.nsw.gov.au/infectious/diseases/pages/covid-19-latest.aspx

${ }^{15}$ URL:https://www.statista.com/statistics/1102730/south-korea-coronavirus-cases-by-age/
} 
расширенными семьями (это может повышать риск инфекции для пожилых). Но, прежде чем пытаться искать подобные объяснения, необходимо понять, как на различия между странами могут влиять подходы к тестированию.

График на рисунке 4, заимствованный с сайта One World in Data, демонстрирует связь между числом тестов и числом подтвержденных случаев заболевания COVID-19 на 20 марта 2020 г. Есть явная корреляция между этими двумя показателями: чем больше делается тестов, тем больше выявленных случаев. Такая корреляция может иметь разные объяснения. С одной стороны, логично предположить, что чем больше страна охвачена инфекцией, тем больше в ней случаев заболевания, и соответственно, тем больше приходится делать тестов; и наоборот, чем меньше заболевших, тем меньше и необходимость в тестах. Но, с другой стороны, эта взаимосвязь может отражать просто тот факт, что чем больше делается тестов, тем больше находится и заболевших.

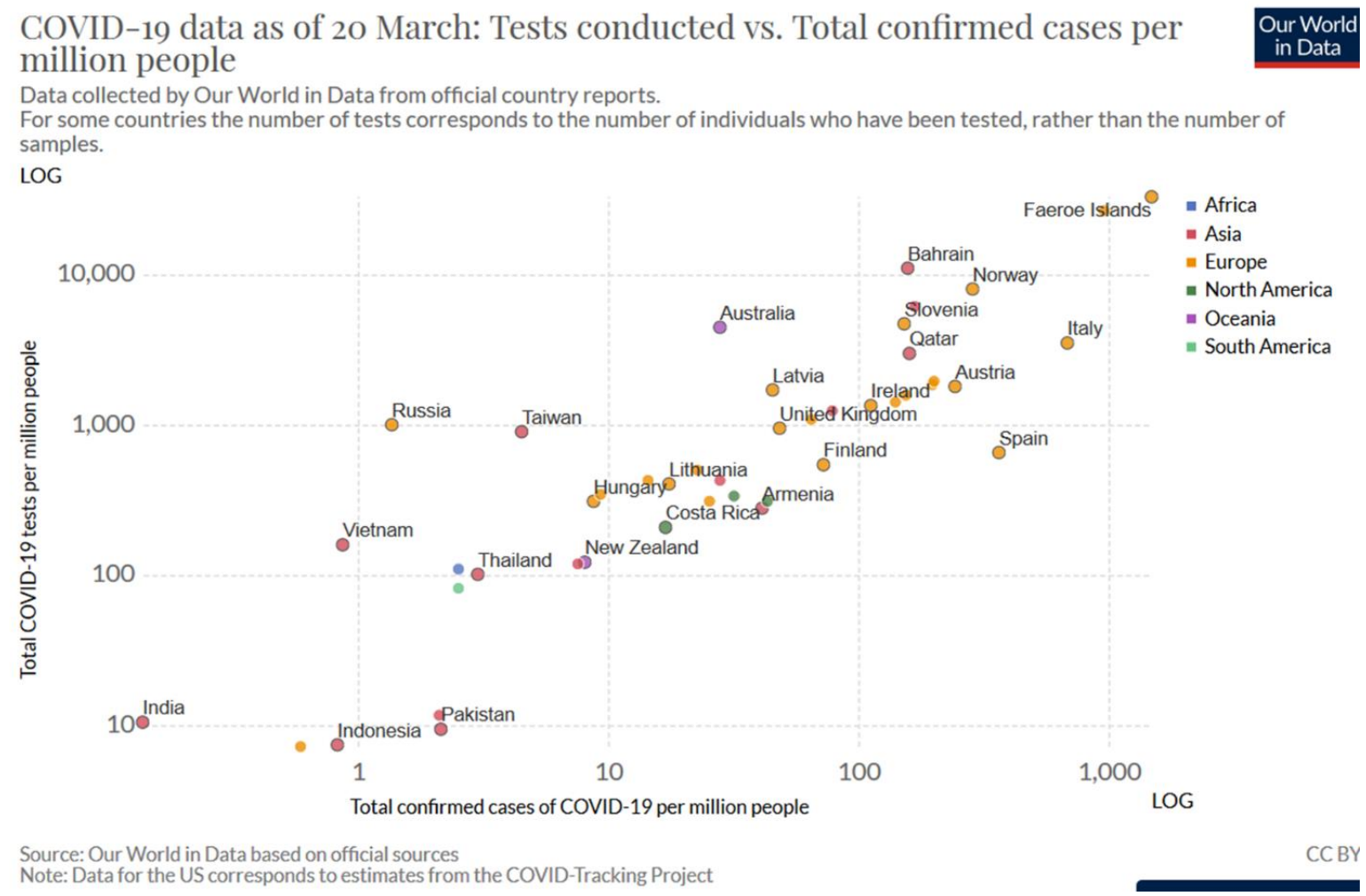

\section{Рисунок 4. Соотношение выполненных тестов на COVID-19 и выявленных случаев заболевания по странам мира}

Источник: Our World in Data. URL:https://ourworldindata.org/coronavirus

На графике видно, что Россия явно выбивается из общего ряда: при сравнительно высоком уровне тестирования у нее относительно низкое число подтвержденных случаев заболевания COVID-19. Также обращают на себя внимание Италия и Испания - страны, которые раньше и сильнее были других поражены инфекцией. Обе страны находятся несколько ниже общего тренда. Это говорит о том, что число проводимых тестов там не так высоко, как можно было бы ожидать из наблюдаемой заболеваемости. 


\section{КАК ОПРЕДЕЛЯЮТ COVID-19 У УМЕРШИХ?}

Теперь коснемся вопроса об учете смертей от коронавируса. Это тоже, конечно, сильно зависит от тестирования. Если прижизненное тестирование выявило у человека COVID-19, то после его смерти мы знаем, что он умер с инфекцией, хотя и это не означает, что он умер именно из-за нее. Но если прижизненного тестирования не было, то мы не знаем, была ли у умершего инфекция, и не факт, что это будет определено. Здесь важное значение может иметь посмертное тестирование, но страны сейчас несколько отличаются в подходах к тому, берут ли они мазки на вирус после смерти или нет.

В США (Centers for Disease Control... 2020) и Великобритании (Osborn et al. 2020) имеющиеся инструкции рекомендуют брать посмертный мазок и тестировать на присутствие вируса в случае, если есть подозрение, что у умершего могла быть инфекция COVID-19. Но эти инструкции носят рекомендательный характер, и решение о том, будет ли выполнен посмертный тест, может приниматься на местах в зависимости от текущих потребностей и ограничений. При этом в обеих странах инструкции допускают запись COVID-19 в свидетельство о смерти без проведения теста в случае, если можно с высокой степенью уверенности полагать, что у умершего был COVID-19 (например, об этом свидетельствуют клинические признаки) (National Vital Statistics... 2020; Office for National Statistics 2020).

Известно, что посмертные тесты делаются также в Италии, Испании, Франции - в тех странах, в которых сейчас наблюдается довольно высокая летальность от COVID-19. Но, например, в Германии «умершие с подозрением на COVID-19, если они не были протестированы при жизни, могут быть протестированы после смерти, но в немецкой децентрализованной системе здравоохранения это не является пока рутинной практикой» ${ }^{16}$.

\section{УМЕРЛИ “ОТ" ИЛИ УМЕРЛИ “С" COVID-19?}

Следующий вопрос: как страны отчитываются о смертях от COVID-19 в случаях, если наряду с коронавирусом к смерти могли привести и другие причины.

Первыми на возможную несопоставимость в этом аспекте между странами обратили внимание итальянские специалисты. Например, A. Borelli, глава итальянского Агентства гражданской защиты, 10 марта, говоря о цифрах смертей COVID-19 в Италии, заявил: «Я бы хотел подчеркнуть, что это смерти не $\boldsymbol{o m}$ коронавируса. Это смерти людей, у которых среди прочих патологий был также и коронавирус» ${ }^{17}$. W. Ricciardi, консультант итальянского Министерства здравоохранения по вопросам COVID-19 и член Европейского совещательного комитета по исследованию в области здоровья (EACHR - одна из структур внутри европейского офиса ВОЗ), 10 марта сказал в интервью итальянскому веб-журналу «Scienza in Rete»: «...мы [в Италии] все смерти записываем в числитель без того

${ }^{16}$ Oltermann P. (2020). Germany's low coronavirus mortality rate intrigues experts. The Guardian. 22.03. URL: https://www.theguardian.com/world/2020/mar/22/germany-low-coronavirus-mortality-rate-puzzles-experts

${ }^{17}$ Come vanno letti i dati sul coronavirus in Italia (2020). Agenzia Italia. 12.03. URL: https://www.agi.it/factchecking/news/2020-03-12/coronavirus-bilancio-morti-contagi-guariti-dati-7447972/ 
маниакального внимания к определению причин смерти, какое есть, например, у французов и немцев, которые, прежде чем констатировать смерть от коронавируса, выполняют серию проверок и оценок, иногда приводящих даже к тому, что смерть исчезает из списка [смертей от коронавируса]» ${ }^{18}$. Его же комментарий приводит The Telegraph 21 марта: «Способ, каким мы кодируем смерти в нашей стране, очень щедрый, в том смысле что все люди, которые умирают в больницах с коронавирусом, считаются умершими от коронавируса. При перепроверке Национальным институтом здоровья только 12\% свидетельств о смерти показали прямую взаимосвязь с коронавирусом, тогда как у 88\% пациентов, которые умерли, было по крайней мере одно предшествующие заболевание, а у многих даже два или три» ${ }^{19}$.

Такой же подход, как в Италии, по всей видимости, принят и в США. D. Birx, ответственный координатор Белого дома США по вопросу коронавируса, сказала на прессконференции 7 апреля: «Есть страны с другим подходом. Если у человека уже было хроническое заболевание и, скажем, вирус заставил его обратиться в отделение интенсивной терапии, а затем у него возникли проблемы с сердцем или почками, - то в некоторых странах смерть в этом случае регистрируется как следствие этих проблем, а не как смерть от COVID-19. Мы сейчас учитываем и намерены... именно сейчас всех, кто умирает с COVID-19, считать умершими от COVID-19» ${ }^{20}$.

Но не во всех странах принят такой подход. Отвечая на запрос Bayerischer Rundfunk о том, кого в Германии считают умершим от COVID-19, пресс-секретарь института Роберта Коха сообщила, что число умерших от COVID-19 включает, в первую очередь, людей, непосредственно умерших от болезни COVID-19 («умершие от») и, во-вторых, пациентов с предшествующими заболеваниями, которые были инфицированы COVID-19 и для которых невозможно однозначно определить что же в конце концов стало причиной смерти («умершие с») $)^{21}$ (Bayerischer Rundfunk 2020). Из данного разъяснения можно предположить, что, если точно доказано, что человек, инфицированный COVID-19, умер от другой причины, то его смерть не попадает в статистику смертей COVID-19 в Германии. И здесь мы, по всей видимости, наблюдаем разницу подходов между Италией и США, с одной стороны, и Германией, с другой.

Рассмотрим ещё два примера.

В Исландии первый случай смерти человека c COVID-19-инфекцией был зафиксирован в середине марта. На сайте исландского Управления здравоохранением ${ }^{22}$

\footnotetext{
${ }^{18}$ Carra L. (2020). Walter Ricciardi: ancora due settimane dure, possibile catastrofe negli USA. Scienza in Rete. 10.03. URL: https://www.scienzainrete.it/articolo/walter-ricciardi-ancora-due-settimane-dure-possibile-catastrofenegli-usa/luca-carra/2020

${ }^{19}$ Newey S. (2020). Why have so many coronavirus patients died in Italy? The Telegraph. 23.03.

URL:https://www.telegraph.co.uk/global-health/science-and-disease/have-many-coronavirus-patients-died-italy/

${ }^{20}$ Remarks by President Trump, Vice President Pence, and Members of the Coronavirus Task Force in Press Briefing (2020). April 7. URL:https://www.whitehouse.gov/briefings-statements/remarks-president-trump-vicepresident-pence-members-coronavirus-task-force-press-briefing-april-7-2020/

${ }^{21}$ Faktenfuchs: Wie werden Corona-Todesfälle gezählt? (2020). Bayerischer Rundfunk. 03.04.

URL:https://www.br.de/nachrichten/deutschland-welt/faktenfuchs-so-werden-corona-todesfaelle-gezaehlt,RtnpYVL

${ }^{22}$ URL:https://www.landlaeknir.is/um-embaettid/greinar/grein/item38863/Stoduskyrslur---Ovissustig-vegnakoronaveiru-(2019-nCoV)
} 
публикуют ежедневные отчеты о ситуации по коронавирусу, в том числе дается информация о числах заболевших, госпитализированных, находящихся в палатах реанимации, умерших. Первое сообщение о смерти появилось 17 марта. Речь шла о туристе из Австралии. Незадолго до смерти он обратился в госпиталь, у него был обнаружен COVID-19, и вскоре после прибытия в госпиталь он скончался. В отчете от 17 марта был подтвержден факт смерти, и при этом подчеркивалось, что причина смерти неизвестна, но симптомы не были типичны для COVID-19 (National Commissioner... 2020a). Далее вплоть до 24 марта в ежедневных отчетах упоминания о смертях нет. Указывается число госпитализированных, число больных, количество палат интенсивной терапии, но случаи смерти не упоминаются. Следующая запись о смертях появилась только 24 марта. К этому времени уже сделали вскрытие умершего австралийца, которое подтвердило, что у него были признаки пневмонии. На тот момент в Исландии умерла еще одна женщина от COVID-19. И только тогда (24 марта) появляется запись, что два человека в Исландии умерли с диагностированным COVID-19 (National commissioner... 2020b). Возникает вопрос: если бы вскрытие туриста из Австралии не показало признаков пневмонии, включили ли бы этот случай в статистику смертей от COVID-19 в Исландии или нет?

Похожий случай был и в России, где первая смерть человека с диагнозом COVID-19 была зафиксирована у женщины в середине марта. Прижизненное тестирование показало, что у неё был COVID-19. Но при вскрытии признаков пневмонии обнаружено не было. О. Зайратьянц, главный патологоанатом Москвы, комментируя результаты вскрытия, сообщил следующее: «На момент смерти изменений в легких не обнаружено, но подтвердились тяжелые сопутствующие хронические заболевания. Среди них сахарный диабет, цереброваскулярная болезнь и ишемическая болезнь сердца» ${ }^{23}$. Насколько можно судить, эта смерть не была включена в статистику случаев смерти от COVID-19 в России.

Таким образом, подходы к тому, как страны отчитываются о смертях от COVID-19, могут различаться. По всей видимости, некоторые страны включают в статистику всех умерших, у кого подтвержден диагноз COVID-19. Другие пытаются сначала определить, мог ли COVID-19 привести к смерти или смерть явилась результатом другой болезни.

Все это относится к цифрам, которые мы сейчас видим на различных сайтах, публикующих числа умерших и заболевших COVID-19. Эти числа собираются с целью глобального наблюдения за эпидемией. Но совсем не обязательно, что такие же числа умерших от этой причины мы позднее увидим в статистике, которая собирается на основе медицинских свидетельств о смерти.

\section{КАК COVID-19 ЗАПИСЫВАЕТСЯ В МЕДИЦИНСКОЕ СВИДЕТЕЛЬСТВО О СМЕРТИ?}

Сейчас ВО3 ввела два новых кода для обозначения COVID-19. Пока это временные коды, которые, видимо, впоследствии попадут в класс респираторных болезней. Речь идет о коде

\footnotetext{
${ }^{23}$ Врачи назвали причиной смерти пациентки с коронавирусом оторвавшийся тромб (2020). Коммерсант. 19.03. URL:https://www.kommersant.ru/doc/4293081
} 
U07.1 (COVID-19, вирус идентифицирован) и коде U07.2 (COVID-19, вирус не идентифицирован). Второй код указывает на случаи COVID-19, которые а) диагностированы клинически и эпидемиологически; б) возможные случаи COVID-19; 3) подозрительные случаи на COVID-19. И код U07.1, и код U07.2 могут записываться в медицинское свидетельство о смерти (World Health Organization 2020d).

При этом в инструкции по кодированию смертей от COVID-19 согласно правилам МКБ-10 ВОЗ отмечает, что COVID-19 указывается в медицинском свидетельстве о смерти так же, как и любая другая причина смерти, и правила для выбора первоначальной причины смерти должны быть, такими же, что и для гриппа. Для этого не нужно никаких специальных инструкций (World Health Organization 2020d) ${ }^{24}$.

В медицинском свидетельстве о смерти есть два блока для записи причин смерти (рисунок 5).

19. Причины смерти:
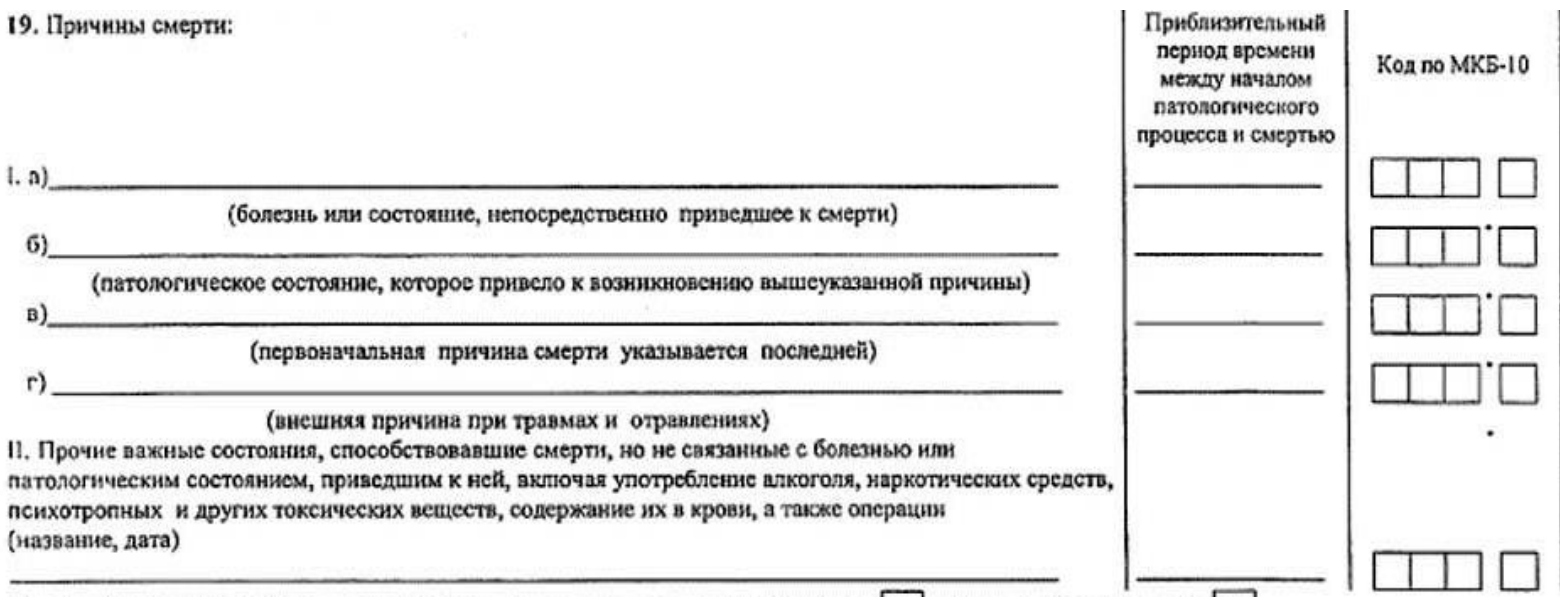

\section{Рисунок 5. Рубрика «Причины смерти» в медицинском свидетельстве о смерти}

Первый пронумерованный блок (I) - это цепь событий, которая непосредственно привела к смерти. Здесь указываются: а) болезненное состояние, непосредственно приведшее к смерти; б) патологическое состояние, которое привело к возникновению вышеуказанной причины смерти; с) первоначальная причина смерти. Согласно определению ВО3, первоначальной причиной смерти считается болезнь или травма, вызвавшая цепь болезненных процессов, непосредственно приведших к смерти (Всемирная организация здравоохранения 1995). Первоначальная причина смерти - это то, чем мы в основном оперируем, когда говорим о причинах смерти в демографическом анализе. То есть у нас во всех базах данных по причинам смерти, например в базе данных ВО3 или Росстата, публикуется статистика именно по первоначальной причине смерти. Кроме блока, где фиксируются состояния, непосредственно приведшие к смерти, в медицинском свидетельстве о смерти есть нижнее поле (блок II), где указываются так называемые сопутствующие причины смерти. Это важные состояния, способствующие

\footnotetext{
2416 апреля 2020 г. ВОЗ все же опубликовала более подробные инструкции о кодировании COVID-19 в медицинском свидетельстве о смерти (World Health Organization 2020e).
} 
смерти, но не связанные с болезнью или патологическим состоянием, указанными в верхнем блоке (блоке I) медицинского свидетельства о смерти.

Какой может быть последовательность событий, чтобы COVID-19 был указан как первоначальная причина смерти? Это, например, случай, когда COVID-19 развивается в пневмонию и затем человек умирает от острого респираторного дистресс-синдрома. При такой последовательности событий определить первоначальную причину смерти легко. Но более сложный вопрос: как кодировать первоначальную причину смерти, если у человека есть сопутствующие хронические заболевания и они обостряются на фоне COVID-19, в результате чего он умирает все же от осложнений своего хронического заболевания?

Если мы обратимся к инструкциям МКБ-10 и к примерам того, как в этом случае надо кодировать грипп, то во втором томе МКБ приведено несколько примеров, как следует выбирать первоначальную причину смерти в случае гриппа (Всемирная организация здравоохранения 1995). Вот один такой пример. Врач, который заполнил медицинское свидетельство о смерти, зафиксировал следующую последовательность событий в верхнем поле рубрики свидетельства «Причины смерти» (блок I): человек умер от острого инфаркта миокарда, который был вызван атеросклеротической болезнью сердца, а она в свою очередь была вызвана гриппом. Грипп записан первоначальной причиной смерти. Согласно правилам выбора первоначальной причины смерти, это пример неправильной последовательности событий, потому что грипп не может вызвать атеросклеротическую болезнь сердца. В данном случае кодировщик или автоматическая система кодирования, обрабатывая медицинское свидетельство смерти, должны исправить последовательность причин смерти и выбрать в качестве первоначальной причиной смерти острый инфаркт миокарда. Грипп в этом случае может быть записан во второй части медицинского свидетельства о смерти как сопутствующая причина.

Подобный же пример приводится в инструкции по кодированию причин смерти Центра по контролю за заболеваниями в США: человек в течение одной недели болеет гриппом, но у него также есть заболевание почек, гипертензия и атеросклероз, хронический диабет. И вот, болея гриппом, он умирает от инфаркта миокарда. Инструкция предписывает, что в этом случае именно инфаркт миокарда должен быть выбран в качестве первоначальной причины смерти (Centers for Disease Control... 2014).

Надо понимать, что если мы говорим, например, о гриппе, то вообще грипп указывается в медицинском свидетельстве о смерти крайне редко. Например, в 2018 г. в качестве первоначальной причины смерти в России он был указан всего 225 раз (коды МКБ-10 J09-J11). Наверное, есть сколько-то еще случаев, когда грипп был указан во второй части медицинского свидетельства как сопутствующая причина смерти. Но в той статистике, которой мы привыкли оперировать, где каждому случаю смерти соответствует одна причина смерти, -225 случаев смертей от гриппа.

Примерно то же самое, например, в Англии. В качестве первоначальной причины смерти грипп в сезоне 2016-2017 (с 1 сентября 2016 г. по 31 августа 2017 г.) годов был зафиксирован 408 раз. Ещё в 571 свидетельстве о смерти грипп был указан в качестве сопутствующей причины смерти (Office for National Statistics 2018). Итого в сезоне 2016- 
2017 гг. в Англии было выписано менее 1000 медицинских свидетельств, в которых было вообще упомянуто о гриппе. При этом, по оценкам английского офиса общественного здравоохранения, в том же сезоне 18009 смертей (средняя оценка) в Англии были ассоциированы с гриппом (Public Health England 2019). Как получена эта оценка? Обычный и потому ожидаемый уровень смертности сопоставляется с наблюдаемым в период эпидемии гриппа и оценивается, насколько этот уровень вырос в результате эпидемии. Таким образом можно определить, сколько дополнительных смертей прибавилось в момент эпидемии гриппа, и, сделав поправку на изменение других условий (например, низкая температура воздуха также приводит к увеличению числа смертей), оценить, сколько «дополнительных» смертей ассоциированы с гриппом.

Если допустить, что внесение COVID-19 в медицинское свидетельство о смерти будет вестись по тем же правилам, что и для гриппа, то можно ожидать, что число смертей от COVID-19, отраженных в статистике, будет меньше, чем те числа смертей, которые сегодня публикуются по странам в целях отслеживания глобального распространения COVID-19. Это верно, по крайней мере, для таких стран, как Италия и США, которые сегодня ведут статистику по смертям «с» COVID-19, а не по смертям «от» COVID-19. С другой стороны, число смертей, «ассоциированных» с COVID-19, которое мы позднее сможем оценить по разнице между ожидаемым и реальным числом смертей в момент эпидемии, будет также существенно отличаться как от числа смертей от COVID-19, которое попадет в статистику на основании первоначальной причины смерти в медицинских свидетельствах о смерти, так и от числа умерших, которые сегодня страны публикуют для целей глобального наблюдения за эпидемией.

\section{ЗАКЛЮЧЕНИЕ}

Данные о числах заболевших и умерших от COVID-19 сегодня собираются странами в оперативном режиме и становятся быстро доступны всем желающим. Открытые данные об эпидемии крайне важны для контроля над заболеванием и для информирования населения. Они используются как исследователями и правительствами для анализа эпидемии и моделирования ее дальнейшего развития, так и средствами массовой информации, блогерами и другими лицами, формирующими общественное мнение. На основе публикуемых чисел о заболеваниях и смертях от COVID-19 делается множество различных выводов, которые быстро распространяются в сети Интернет через социальные медиаплатформы. Но, несмотря на доступность и открытость данных, которые существуют сегодня, гораздо меньше известно о том, на основании каких критериев эти данные собираются и в чем их ограничения. Анализ доступной информации о том, как числа заболевших и умерших собираются в разных странах, приведенный в данной статье, показывает, что данные могут быть несопоставимы между странами. Страны используют различные критерии как для проведения тестов на вирус и выявления заболевших, так и для определения смертей от COVID-19. Эти критерии могут также меняться и во времени. Непонимание ограничений данных, степени их сопоставимости может привести к ложным выводам и интерпретациям. Сравнение стран по заболеваемости и летальности от COVID19 на основе данных, собираемых согласно разным критериям, может иметь и далеко 
идущие политические последствия: как, исходя из данной информации, люди оценят усилия, предпринятые правительством их страны по сокращению заболеваемости и смертности? Поэтому, информируя население о количестве случаев заболеваний и смертей от COVID-19, приводя статистику для различных территорий, не менее важно также четко и ясно сообщать, что стоит за этими данными и в чем их ограничения.

\section{ЛИТЕРАТУРА}

Australian National University (2020). Australian Human Mortality Database. Retrieved from https://demography.cass.anu.edu.au/research/australian-human-mortality-database/dataexplanation (data downloaded on 30.03.2020).

Centers for Disease Control and Prevention (2014). ICD-10-Mortality Manual $2 a$ - 2014. Retrieved from: https://www.cdc.gov/nchs/data/dvs/2a_2014.pdf

Centers for Disease Control and Prevention (2020). Collection and Submission of Postmortem Specimens from Deceased Persons with Known or Suspected COVID-19, March 2020 (Interim Guidance). Retrieved from: https://www.cdc.gov/coronavirus/2019ncov/hcp/guidance-postmortem-specimens.html

Gudbjartsson D.F., Helgason A., Jonsson H., Magnusson O., Melsted P., Norddahl G.L., Saemundsdottir J. et al. (2020). Spread of SARS-CoV-2 in the Icelandic Population. The New England Journal of Medicin. DOI: 10.1056/NEJMoa2006100

University of California, Berkeley, Max Planck Institute for Demographic Research (2020). The Human Mortality Database. Retrieved from http://www.mortality.org/ (data downloaded on 30.03.2020).

National Commissioner of the Icelandic Police (2020a). Status Report 17.03.2020. Retrieved from:

https://www.landlaeknir.is/servlet/file/store93/item39951/COVID19_Status_Report_170320 20.pdf

National Commissioner of the Icelandic Police (2020b). Status Report 24.03.2020. Retrieved from: https://www.landlaeknir.is/servlet/file/store93/item40626/COVID19_Stoduskyrsla_2403202 0.pdf

National Vital Statistics Service (2020). Guidance for Certifying Deaths Due to Coronavirus Disease 2019 (COVID-19). Retrieved from: https://www.cdc.gov/nchs/data/nvss/vsrg/vsrg03-508.pdf

Office for National Statistics (2018). Deaths where the underlying cause or contributory cause was flu, by age group, England, year ending 2013 to year ending 2017 flu year occurrences. Retrieved from:

https://www.ons.gov.uk/peoplepopulationandcommunity/birthsdeathsandmarriages/deaths/ad hocs/009384deathswheretheunderlyingcauseorcontributorycausewasflubyagegroupenglandye arending2013toyearending2017fluyearoccurrences

Office for National Statistics (2020). Guidance for doctors completing Medical Certificates of Cause of Death in England and Wales. Retrieved from: https://assets.publishing.service.gov.uk/government/uploads/system/uploads/attachment_data /file/877302/guidance-for-doctors-completing-medical-certificates-of-cause-of-death-covid19.pdf 
Osborn M., Lucas S., Stewart R., Swift B., Youd E. Briefing on COVID-19. Autopsy practice relating to possible cases of COVID-19 (2019-nCov, novel coronavirus from China 2019/2020). Retrieved from: https://www.rcpath.org/uploads/assets/d5e28baf-5789-4b0facecfe370eee6223/fe8fa85a-f004-4a0c-81ee4b2b9cd12cbf/Briefing-on-COVID-19-autopsyFeb-2020.pdf

Public Health England (2019). Surveillance of influenza and other respiratory viruses in the UK Winter 2018to 2019. Retrieved from:

https://assets.publishing.service.gov.uk/government/uploads/system/uploads/attachment_data /file/839350/Surveillance_of_influenza_and_other_respiratory_viruses_in_the_UK_2018_to _2019-FINAL.pdf

Robert Koch Institut (2020a). 2019-nCoV: Verdachtsabklärung und Maßnahmen. Orientierungshilfe für Ärztinnen und Ärzte. 21.01.2020. doi: 10.25646/6454

Robert Koch Institut (2020b). 2019-nCoV: Verdachtsabklärung und Maßnahmen. Orientierungshilfe für Ärztinnen und Ärzte. 23.01.2020. doi: 10.25646/6454.2

Robert Koch Institut (2020c). 2019-nCoV: Verdachtsabklärung und Maßnahmen. Orientierungshilfe für Ärztinnen und Ärzte. 10.02.2020. doi: 10.25646/6473

Robert Koch Institut (2020d). COVID-19: Verdachtsabklärung und Maßnahmen. Orientierungshilfe für Ärztinnen und Ärzte. 26.02.2020. doi: 10.25646/6473.3

Robert Koch Institut (2020e). COVID-19: Verdachtsabklärung und Maßnahmen. Orientierungshilfe für Ärztinnen und Ärzte. 24.03.2020. doi: 10.25646/6473.7

Santé publique France (2020). COVID-19 Point épidémiologique hebdomadaire du 2 avril 2020. Retrieved from: https://www.santepubliquefrance.fr/maladies-et-traumatismes/maladies-etinfections-respiratoires/infection-a-coronavirus/documents/bulletin- national/COVID-19point-epidemiologique-du-2-avril-2020

World Health Organization (2020a). WHO Director-General's opening remarks at the media briefing on COVID-19 - 11 March 2020. Retrieved from https://www.who.int/dg/speeches/detail/who-director-general-s-opening-remarks-at-themedia-briefing-on-COVID-19---11-march-2020

World Health Organization (2020b). Global surveillance for COVID-19caused by human infection with COVID-19 virus. Interim guidance. 20 March 2020. Retrieved from https://apps.who.int/iris/bitstream/handle/10665/331506/WHO-2019-nCoVSurveillanceGuidance-2020.6-eng.pdf

World Health Organization (2020c). Laboratory testing strategy recommendations for COVID19. Interim guidance. 21 March 2020. Retrieved from: https://apps.who.int/iris/bitstream/handle/10665/331509/WHO-COVID-19-lab_testing2020.1-eng.pdf

World Health Organization (2020d). COVID-19 coding in ICD-10. Retrieved from: https://www.who.int/classifications/icd/COVID-19-coding-icd10.pdf?ua=1

World Health Organization (2020e). International guidelines for certification and classification (coding) of COVID-19 as cause of death. Retrieved from: https://www.who.int/classifications/icd/Guidelines_Cause_of_Death_COVID-19.pdf

Всемирная организация здравоохранения (1995). МКБ-10: Международная статистическая классификачия болезней и проблем, связанных со здоровьем. Десятый пересмотр. Женева: Всемирная Организация Здравоохранения. 


\title{
MORBIDITY AND MORTALITY FROM COVID-19. THE PROBLEM OF DATA COMPARABILITY
}

\section{INNA DANILOVA}

\begin{abstract}
The numbers of COVID-19 cases and deaths are currently attracting worldwide attention. Different web resources publish these data for countries and their regions. The data are available to everyone and are updated in almost real-time mode. The rapid collection of data on cases and deaths and the open access of these data clearly provide important advantages in combating the epidemic, predicting its spread, and planning containment measures. An important area of research on the COVID-19 epidemic is the comparison of countries and territories by their levels of morbidity, lethality, and mortality. Given the opportunities opening up today for data analysis, it is crucially important to understand according to what criteria the data are collected.
\end{abstract}

This paper discusses the key issues of collecting data on COVID-19 cases and deaths and raises the question of data comparability across countries. It shows that countries differ significantly in their approaches to reporting COVID-19 cases and deaths. The data comparability across countries can be influenced, among other things, by the availability of tests, the criteria adopted by the country for testing for the virus (both while the patient is alive and post-mortem), and approaches to determining the cause of death and recording COVID-19 deaths in those with pre-existing chronic conditions. Different approaches to recording COVID19 cases and deaths across countries, as well as their changes over time, pose significant limitations to our ability to assess the spread of the epidemic. These limitations should be taken into account when performing the analysis of COVID-19 morbidity and mortality. Ignoring these limitations can significantly distort our understanding of the spread of the epidemic across different territories.

Key words: COVID-19, data collection, causes of death, morbidity statistics, mortality statistics.

InNa Danilova (danilova@demogr.mpg.de), MAX Planck Institute For DEMOGRAPHic RESEARCh, GERMANY.

DATE RECEIVED : APRIL 2020.

\section{REFERENCES}

Australian National University (2020). Australian Human Mortality Database. Retrieved from https://demography.cass.anu.edu.au/research/australian-human-mortality-database/dataexplanation (data downloaded on 30.03.2020).

Centers for Disease Control and Prevention (2014). ICD-10-Mortality Manual $2 a$ - 2014. Retrieved from: https://www.cdc.gov/nchs/data/dvs/2a_2014.pdf

Centers for Disease Control and Prevention (2020). Collection and Submission of Postmortem Specimens from Deceased Persons with Known or Suspected COVID-19, March 2020 (Interim Guidance). Retrieved from: https://www.cdc.gov/coronavirus/2019ncov/hcp/guidance-postmortem-specimens.html

Gudbjartsson D.F., Helgason A., Jonsson H., Magnusson O., Melsted P., Norddahl G.L., Saemundsdottir J. et al. (2020). Spread of SARS-CoV-2 in the Icelandic Population. The New England Journal of Medicin. DOI: 10.1056/NEJMoa2006100

National Commissioner of the Icelandic Police (2020a). Status Report 17.03.2020. Retrieved from: 
https://www.landlaeknir.is/servlet/file/store93/item39951/COVID19_Status_Report_170320 20.pdf

National Commissioner of the Icelandic Police (2020b). Status Report 24.03.2020. Retrieved from:

https://www.landlaeknir.is/servlet/file/store93/item40626/COVID19_Stoduskyrsla_2403202 $0 . p d f$

National Vital Statistics Service (2020). Guidance for Certifying Deaths Due to Coronavirus Disease 2019 (COVID-19). Retrieved from:

https://www.cdc.gov/nchs/data/nvss/vsrg/vsrg03-508.pdf

Office for National Statistics (2018). Deaths where the underlying cause or contributory cause was flu, by age group, England, year ending 2013 to year ending 2017 flu year occurrences. Retrieved from:

https://www.ons.gov.uk/peoplepopulationandcommunity/birthsdeathsandmarriages/deaths/ad hocs/009384deathswheretheunderlyingcauseorcontributorycausewasflubyagegroupenglandye arending2013toyearending2017fluyearoccurrences

Office for National Statistics (2020). Guidance for doctors completing Medical Certificates of Cause of Death in England and Wales. Retrieved from:

https://assets.publishing.service.gov.uk/government/uploads/system/uploads/attachment_data /file/877302/guidance-for-doctors-completing-medical-certificates-of-cause-of-death-covid19.pdf

Osborn M., Lucas S., Stewart R., Swift B., Youd E. Briefing on COVID-19. Autopsy practice relating to possible cases of COVID-19 (2019-nCov, novel coronavirus from China 2019/2020). Retrieved from: https://www.rcpath.org/uploads/assets/d5e28baf-5789-4b0facecfe370eee6223/fe8fa85a-f004-4a0c-81ee4b2b9cd12cbf/Briefing-on-COVID-19-autopsyFeb-2020.pdf

Public Health England (2019). Surveillance of influenza and other respiratory viruses in the UK Winter 2018to 2019. Retrieved from:

https://assets.publishing.service.gov.uk/government/uploads/system/uploads/attachment_data /file/839350/Surveillance_of_influenza_and_other_respiratory_viruses_in_the_UK_2018_to _2019-FINAL.pdf

Robert Koch Institut (2020a). 2019-nCoV: Verdachtsabklärung und Maßnahmen. Orientierungshilfe für Ärztinnen und Ärzte. 21.01.2020. doi: 10.25646/6454

Robert Koch Institut (2020b). 2019-nCoV: Verdachtsabklärung und Maßnahmen. Orientierungshilfe für Ärztinnen und Ärzte. 23.01.2020. doi: 10.25646/6454.2

Robert Koch Institut (2020c). 2019-nCoV: Verdachtsabklärung und Maßnahmen. Orientierungshilfe für Ärztinnen und Ärzte. 10.02.2020. doi: 10.25646/6473

Robert Koch Institut (2020d). COVID-19: Verdachtsabklärung und Maßnahmen. Orientierungshilfe für Ärztinnen und Ärzte. 26.02.2020. doi: 10.25646/6473.3

Robert Koch Institut (2020e). COVID-19: Verdachtsabklärung und Maßnahmen. Orientierungshilfe für Ärztinnen und Ärzte. 24.03.2020. doi: 10.25646/6473.7

Santé publique France (2020). COVID-19 Point épidémiologique hebdomadaire du 2 avril 2020. Retrieved from: https://www.santepubliquefrance.fr/maladies-et-traumatismes/maladies-etinfections-respiratoires/infection-a-coronavirus/documents/bulletin- national/COVID-19point-epidemiologique-du-2-avril-2020 
University of California, Berkeley, Max Planck Institute for Demographic Research (2020). The Human Mortality Database. Retrieved from http://www.mortality.org/ (data downloaded on 30.03.2020).

Vsemirnaya organizatsiya zdravookhraneniya (1995). MKB-10: Mezhdunarodnaya statisticheskaya klassifikatsiya bolezney i problem, svyazannykh so zdorov'yem. Desyatyy peresmotr [ICD-10: international statistical classification of diseases and health-related problems. Tenth revision]. Geneva: World Health Organization. (In Russ.).

World Health Organization (2020a). WHO Director-General's opening remarks at the media briefing on COVID-19 - 11 March 2020. Retrieved from https://www.who.int/dg/speeches/detail/who-director-general-s-opening-remarks-at-themedia-briefing-on-COVID-19---11-march-2020

World Health Organization (2020b). Global surveillance for COVID-19caused by human infection with COVID-19 virus. Interim guidance. 20 March 2020. Retrieved from https://apps.who.int/iris/bitstream/handle/10665/331506/WHO-2019-nCoVSurveillanceGuidance-2020.6-eng.pdf

World Health Organization (2020c). Laboratory testing strategy recommendations for COVID19. Interim guidance. 21 March 2020. Retrieved from: https://apps.who.int/iris/bitstream/handle/10665/331509/WHO-COVID-19-lab_testing2020.1-eng.pdf

World Health Organization (2020d). COVID-19 coding in ICD-10. Retrieved from: https://www.who.int/classifications/icd/COVID-19-coding-icd10.pdf?ua=1

World Health Organization (2020e). International guidelines for certification and classification (coding) of COVID-19 as cause of death. Retrieved from:

https://www.who.int/classifications/icd/Guidelines_Cause_of_Death_COVID-19.pdf 